\title{
Knockdown of STIP1 inhibits the invasion of CD133-positive cancer stem-like cells of the osteosarcoma MG63 cell line via the PI3K/Akt and ERK1/2 pathways
}

\author{
JIAN-HUA WANG $^{1 *}$, CHEN GONG $^{1 *}$, FENG-JIN GUO $^{2}$, XIAO ZHOU $^{1}$, MING-SHENG ZHANG $^{1}$, \\ HONG QIU $^{1}$, TENG-FEI CHAO ${ }^{1}$, YANG LIU ${ }^{2}$, LIANG QIN $^{2}$ and HUI-HUA XIONG ${ }^{1}$ \\ Departments of ${ }^{1}$ Oncology and ${ }^{2}$ Orthopedics, Tongji Hospital, Tongji Medical College, \\ Huazhong University of Science and Technology, Wuhan, Hubei 430030, P.R. China
}

Received March 23, 2019; Accepted July 24, 2019

DOI: $10.3892 /$ ijmm.2020.4764

\begin{abstract}
Osteosarcoma is the most common primary malignant tumor of the bone in adolescents and children, with high rates of metastasis and a poor prognosis. Recently, osteosarcoma cancer stem/stem-like cells (CSCs) have been identified as the main cause of recurrence and metastasis. Stress-induced phosphoprotein 1 (STIP1), a co-chaperone that binds to heat shock proteins 70 and 90 , is abnormally expressed in several tumor cell lines, and may play an important role in tumor cell migration and invasion. These features indicate that STIP1 may represent a new therapeutic target for osteosarcoma CSCs. However, the role of STIP1 in osteosarcoma CSC migration and invasion remains largely unknown. In the present study, CD133-positive osteosarcoma CSCs were first isolated and cultured by magnetic cell sorting and serum-free medium suspension cell sphere culture, respectively. Knockdown of STIP1 by small interfering RNA significantly was then shown to inhibit the migration and invasion of these cells, possibly due to the regulation of the expression of matrix metalloproteinase (MMP)-2, MMP-9 and tissue inhibitor of metalloproteinase-2. Furthermore, data from the present study suggested that the
\end{abstract}

Correspondence to: Professor Hui-Hua Xiong, Department of Oncology, Tongji Hospital, Tongji Medical College, Huazhong University of Science and Technology, 1095 Jie Fang Avenue, Wuhan, Hubei 430030, P.R. China

E-mail: xionghuihua@hotmail.com

${ }^{*}$ Contributed equally

Abbreviations: STIP1, stress-induced phosphoprotein 1; HSP heat shock proteins; HOP, HSP70/HSP90 organizing protein; CSCs, cancer stem/stem-like cells; p-Akt, phosphorylated Akt; MMP, matrix metalloproteinase; TIMP, tissue inhibitor of metalloproteinase; siRNA, small interfering RNA; ERK1/2, extracellular regulated protein kinases $1 / 2$

Key words: osteosarcoma, CSCs, STIP1, ERK1/2 pathway, PI3K/Akt pathway knockdown of STIP1 decreased the levels of phosphorylated Akt and phosphorylated ERK1/2. In summary, these findings indicate that targeting STIP1 in osteosarcoma may constitute a viable molecular targeted therapy strategy for the inhibition of CSC invasion and migration.

\section{Introduction}

Osteosarcoma is a common primary malignant tumor derived from the musculoskeletal system with high rates of recurrence and metastasis. Therapeutic options have significantly improved over time, especially in terms of chemotherapy and limb-sparing surgery. The 5-year survival rate of patients with osteosarcoma increased from 20 to $70-80 \%$ after the introduction of neo-adjuvant chemotherapy (1). However, to the best of our knowledge, further improvements to the 5-year survival rate have not been reported for nearly 30 years, mainly due to distant metastasis and recurrence. Conventional treatments used for advanced osteosarcoma, such as surgery, chemotherapy, radiotherapy or combinations of these, have had little effect on the long-term survival of patients with this disease (2). New drugs are needed in order to improve the outcomes of advanced osteosarcoma.

Molecular chaperones are a class of proteins that mediate the assembly or disassembly of other macromolecular structures through catalytic or non-catalytic routes. Heat shock proteins (HSPs) are the major type of chaperones, which are expressed in response to elevated temperatures or other cellular stresses and prevent or correct damage caused by heat-related misfolding (3). HSPs, particularly HSP70 and HSP90, play an important role in cancer-related molecular events by controlling kinases, signaling proteins, cell-cycle regulators and steroid receptors (4). Stress-induced phosphoprotein 1 (STIP1/STI1 or p60), also called HSP70/HSP90 organizing protein $(\mathrm{HOP})$, is a $66-\mathrm{kDa}$ protein and the most extensively studied multi-domain co-chaperone. STIP1, which mediates the activity of the HSP70/HSP90 chaperone heterocomplex, contains a nuclear localization signal regulated by cell cycle kinase phosphorylation, and undergoes nuclear-cytoplasmic shuttling considered to be influenced by cell cycle status and cell cycle kinases $(5,6)$. Compared with normal cells, cancer 
cells can sustain higher metabolic rates through multiple intrinsic mechanisms and signal transduction pathways. The role of STIP1 in cancer remains unclear, but there is increasing evidence that STIP1 may regulate multiple cancer-related biological processes, including tumor formation, metastasis and drug resistance in many types of cancer cells such as pancreatic cancer and gastric cancer (7-9).

A possible mechanism underlying tumor formation, multidrug resistance, recurrence and metastasis is suggested by the cancer stem/stem-like cells (CSCs) theory, according to which a sub-population of cancer cells with self-renewal and differentiation capacities drive tumor proliferation (10). Previous studies have demonstrated that there is a group of CSCs among various cancer cell types, including melanoma, hematopoietic, brain, breast, pancreatic, colon and prostate cancer (10,11). Gibbs et al (12) isolated and identified CSCs from osteosarcoma cells obtained from primary biopsies by using the cell-sphere formation method in serum-free suspension medium containing growth factors. Human osteosarcoma cell lines contain CD133-positive $\left(\mathrm{CD} 133^{+}\right)$CSC populations, which exhibit increased production of ATP and lactate dehydrogenase under hypoxia, and have high chemoresistance and metastatic potential (13-16). Li et al (17) isolated CD133+ osteosarcoma cells, which exhibited similar properties to CSCs, from the MG63 cell line by magnetic activated cell sorting (MACS).

In the present study, CD133+ ${ }^{+}$CSCs were isolated and identified from the human osteosarcoma MG63 cell line. Furthermore, RNA interference technology was used to knock down STIP1 expression in CSCs isolated from the MG63 cells, and to examine the molecular mechanisms involved in osteosarcoma CSC invasion and migration.

\section{Materials and methods}

Cell lines and culture. The human osteosarcoma MG63 cell line was purchased from the China Center for Type Culture Collection at Wuhan University. Cells were cultured in DMEM/F12 (HyClone; GE Healthcare Life Sciences) supplemented with $10 \%$ FBS (Gibco; Thermo Fisher Scientific, Inc.) in a $5 \% \mathrm{CO}_{2}$ humidified incubator at $37^{\circ} \mathrm{C}$. Cells were digested with $2.5 \mathrm{~g} / \mathrm{l}$ trypsin (Gibco; Thermo Fisher Scientific, Inc.) for 3-5 min at $37^{\circ} \mathrm{C}$ and passaged once every 5-6 days.

Isolation of CSCs from the osteosarcoma MG63 cell line. The process of osteosarcoma CSC isolation was conducted as reported previously $(12,13,18,19)$. MG63 cells were harvested by trypsinization, detached, centrifuged for $5 \mathrm{~min}$ at 1,200 $\mathrm{x}$ g at room temperature and resuspended in $1 \mathrm{ml}$ PBS (cat. no. PYG0021; Wuhan Boster Biological Technology, Ltd.) containing 1\% BSA (cat. no. AR1006; Wuhan Boster Biological Technology, Ltd.) at $1 \times 10^{7}$ cells per $500 \mu 1$, then incubated with an anti-CD133 antibody (cat. no. sc-30220; 1:200; Santa Cruz Biotechnology, Inc.) at $37^{\circ} \mathrm{C}$ for $15 \mathrm{~min}$. After two washes with PBS containing $1 \% \mathrm{BSA}$, the cells were labeled with micro-beads (Miltenyi Biotec $\mathrm{GmbH}$ ) at $37^{\circ} \mathrm{C}$ for $15 \mathrm{~min}$. The cells were resuspended and poured into a separation column (LS Columns; Miltenyi Biotec $\mathrm{GmbH}$ ), after which $\mathrm{CD}_{133}{ }^{+}$cells were retained in the column and flushed out with $2 \mathrm{ml}$ PBS containing 1\% BSA with the aid of a plunger.
Subsequently, the $\mathrm{CD}_{133^{+}}$cells were plated in ultra-low-attachment culture plates (Corning Life Sciences) in serum-free medium, consisting of DMEM/F12, $20 \mu \mathrm{g} / 1$ human epidermal growth factor (PeproTech, Inc.), 1X N2 supplement (Invitrogen; Thermo Fisher Scientific, Inc.), $2 \mathrm{mmol} / 1 \mathrm{~L}$-glutamine, $4 \mathrm{U} / 1$ insulin (both Sigma-Aldrich; Merck KGaA), $1 \times 10^{5} \mathrm{U} / 1$ penicillin and $1 \times 10^{5} \mathrm{U} / 1$ streptomycin. The $\mathrm{pH}$ value was adjusted to 7.2-7.5. Additional fresh serum-free medium was added once every other day. After CD133 ${ }^{+}$CSC spheres that contained $>50$ cells formed, the $\mathrm{CD}_{133}{ }^{+} \mathrm{CSC}$ s were collected and dissociated by trypsinization, then passaged in serum-free medium at a ratio of 1:2-1:4.

Flow cytometry analysis. CD133 ${ }^{+}$CSCs were resuspended to single-cell suspensions, washed twice with PBS, and incubated with antibodies against CD44 (cat. no. sc-65265; 1:100; Santa Cruz Biotechnology, Inc.), CD105 (cat. no. sc-53999; 1:100; Santa Cruz Biotechnology, Inc.) and CD133 (1:100) in $0.5 \% \mathrm{BSA}$ and PBS for $60 \mathrm{~min}$ at $4^{\circ} \mathrm{C}$. After washing, samples were incubated with a secondary antibody $(1: 150$; Goat Anti-Mouse IgG H+L Secondary Antibody-FITC Conjugate for CD44 and CD105; cat. no. BA1101; Goat Anti-Rabbit IgG H+L Secondary Antibody-FITC Conjugate for CD133; cat. no. BA1105, Wuhan Boster Biological Technology, Ltd.), in $0.5 \%$ BSA and PBS for $15 \mathrm{~min}$ at $37^{\circ} \mathrm{C}$. Finally, the samples were analyzed by flow cytometry using FACSort with CellQuest, ModFit LT for Mac V2.0 software (BD Biosciences) after washing twice. Each experiment was performed in triplicate.

Transient transfections with small interfering RNA (siRNA). $\mathrm{CD} 133{ }^{+} \mathrm{CSC}$ were seeded into 6-well plates at $4 \times 10^{4}$ cells/well. Three pre-designed siRNAs (Shanghai GenePharma Co., Ltd.) were chosen for the knockdown of STIP1 (STIP1-homo-643, STIP1-homo-941, STIP1-homo-1315) in addition to the negative control (NC) siRNA (Table I). After overnight culture, the medium was changed and the cells were transfected with siRNA or NC, each at a final volume of $5 \mu$ l Lipofectamine ${ }^{\circledR} 2000$ (Invitrogen; Thermo Fisher Scientific, Inc.) and final concentration of $250 \mathrm{pmol}$ siRNA in a total volume of $500 \mu \mathrm{l}$ Opti-MEM (Gibco; Thermo Fisher Scientific, Inc.). All procedures were performed according to the Lipofectamine ${ }^{\circledR} 2000$ manufacturer's protocol. The transfection efficiency was detected by fluorescence microscopy (Olympus BX53) after 24-h culture. Cells cultured for 48-72 h were trypsinized and used in subsequent experiments.

Reverse transcription-quantitative PCR (RT-qPCR) analysis. After the transfection of siRNA for $72 \mathrm{~h}$, total RNA was isolated from the cells using TRIzol ${ }^{\mathrm{TM}}$ (Invitrogen; Thermo Fisher Scientific, Inc.) according to the manufacturer's protocol. The RNA was quantified by spectrophotometry at an optical density of $260 \mathrm{~nm}$. First-strand cDNA was generated from $2 \mu \mathrm{g}$ total RNA using a cDNA Synthesis kit (Invitrogen; Thermo Fisher Scientific, Inc.), according to the manufacturer's protocol, in a total reaction volume of $2 \mu \mathrm{l}$. The primer sequences were obtained from qPrimerDepot (http://primerdepot.nci.nih.gov/) and PrimerBank (http://pga.mgh.harvard.edu/primerbank/), purchased from Invitrogen; Thermo Fisher Scientific, Inc., and are listed in Table II. GAPDH was used as the reference gene. 
Table I. Sequences of the siRNAs used in the present study.

SiRNA

STIP1-homo-643

STIP1-homo-941

STIP1-homo-1315

Negative control
Sequence $\left(5^{\prime}-3^{\prime}\right)$

Sense: GGGCACGAAACUACAAGAUTT

Antisense: AUCUUGUAGUUUCGUGCCCTT

Sense: GCAGCGGUAUACUUUGAAATT

Antisense: UUUCAAAGUAUACCGCUGCTT

Sense: CCCGAAAGAUGCCAAAUUATT

Antisense: UAAUUUGGCAUCUUUCGGGTT

Sense: UUCUCCGAACGUGUCACGUTT

Antisense: ACGUGACACGUUCGGAGAATT

siRNA, small interfering RNA; STIP1, stress-induced phosphoprotein 1.

Table II. Sequences of primers used in the present study.

\begin{tabular}{llc}
\hline Gene & \multicolumn{1}{c}{ Primer sequence (5'-3') } & $\begin{array}{c}\text { Product } \\
\text { length, bp }\end{array}$ \\
\hline STIP1 & F: AACGCGGAGCGGACGGATTC & 101 \\
& R: ACGCTCAGGGCCTTGTTGCC & \\
GAPDH & F: CAATGACCCCTTCATTGACC & 106 \\
& R: GACAAGCTTCCCGTTCTCAG & \\
\hline
\end{tabular}

STIP1, stress-induced phosphoprotein 1 .

qPCR was carried out using the SYBR Green qPCR Master mix (2X; Roche Diagnostics) and an iQ Real-Time system (Bio-Rad Laboratories, Inc.) equipped with a 96-well optical reaction plate in a total reaction volume of $20 \mu \mathrm{l}$. All qPCR reactions were run in triplicate, and the mean threshold cycle value was used for the determination of mRNA levels. The thermocycling conditions were as follows: Initial denaturation step at $95^{\circ} \mathrm{C}$ for $5 \mathrm{~min}$, followed by 40 cycles at $94^{\circ} \mathrm{C}$ for $20 \mathrm{sec}$, $58^{\circ} \mathrm{C}$ for $20 \mathrm{sec}$ and $72^{\circ} \mathrm{C}$ for $20 \mathrm{sec}$, and a final extension step at $72^{\circ} \mathrm{C}$ for $5 \mathrm{~min}$. At the end of the reactions, the baseline and threshold values for the amplifications were set using iCycler5 software version 3.1 (Bio-Rad Laboratories, Inc.), and the relative expression of mRNA was calculated using the comparative $2^{-\Delta \Delta \mathrm{Cq}}$ method (20).

Cell proliferation assays. $\mathrm{CD}_{133}{ }^{+} \mathrm{CSC}$ s were seeded into 96-well plates at a density of 5,000 cells/well in $100 \mu 1$ DMEM/F12 supplemented with $10 \%$ FBS. Cell proliferation was detected 24 and $48 \mathrm{~h}$ after transfection, using the Cell Counting Kit-8 (CCK-8; Beyotime Institute of Biotechnology). A total of $10 \mu \mathrm{l}$ CCK- 8 reagent was added to each well on the 96-well plate, which was then placed in a $\mathrm{CO}_{2}$ incubator for $2 \mathrm{~h}$ to react. Following incubation, the absorbance was measured on a plate reader using a $450 \mathrm{~nm}$ filter. Each assay was performed in six replicates.

Wound-healing assays. CD133+ CSCs were seeded into 6-well plates using DMEM/F12 supplemented with 10\% FBS and formed evenly distributed monolayers. Then the medium was replaced with serum-free DMEM/F12 medium and serum starvation continued overnight. The monolayers were mechanically scratched using a pipette tip to create a wound under the guidance of a sterilized ruler. Cells were washed twice with PBS and fresh serum-free DMEM/F12 medium was added in order to observe wound healing. Each assay was performed in six replicates. Images were captured 24 and 48 h after incubation under an Olympus BX53 light microscope (Olympus Corporation) at $\mathrm{x} 100$ magnification The relative wound distance was calculated as the percentage of the distance in different groups at 24 and $48 \mathrm{~h}$ to the corresponding distance at $0 \mathrm{~h}(21)$.

Matrigel invasion assays. The upper chambers of Transwell plates were coated with $100 \mu \mathrm{l}$ Matrigel (BD Biosciences), then the $\mathrm{CD}_{133}{ }^{+} \mathrm{CSC}$ (NC group and STIP1-siRNA group) at the cell density of $1 \times 10^{5}$ cells/well were added to the upper chambers in $200 \mu \mathrm{l}$ serum-free medium, placed in a 24 -well plate, and $500 \mu \mathrm{l}$ DMEM/F12 supplemented with $10 \%$ FBS was added to the lower chambers. Five replicates were performed for each group. Following $24 \mathrm{~h}$ incubation at $37^{\circ} \mathrm{C}$, the non-invading cells that remained on the upper membranes were removed by swabbing. The invading cells on the underside of the filters were fixed with $4 \%$ paraformaldehyde for $30 \mathrm{~min}$, stained with $0.1 \%$ crystal violet for $10 \mathrm{~min}$ at room temperature, and then images were captured under light microscopy (magnification, x200) for quantification. The extent of cell invasion was quantified as the number of invading cells in the STIP1-siRNA group divided by the number of invading cells in the NC group.

Western blot analysis. Briefly, total protein was extracted from cells using RIPA lysis buffer containing a freshly added proteinase inhibitor cocktail and a phosphatase inhibitor cocktail (all Wuhan Boster Biological Technology, Ltd.). Protein concentrations were quantified using a BCA Protein Assay kit (Thermo Fisher Scientific, Inc.). Protein samples were denatured at $100^{\circ} \mathrm{C}$ for $10 \mathrm{~min}$ and then preserved at $-20^{\circ} \mathrm{C}$ for later use. The proteins $(50 \mu \mathrm{g} /$ lane $)$ were separated via SDS-PAGE (12\% gel), and then transferred onto polyvinylidene fluoride (PVDF) membranes (Millipore), which were treated with TBS-0.05\% Tween-20 (TBS-T) blocking buffer (cat. no. AR0179; Wuhan Boster Biological Technology, Ltd.) 
for $1 \mathrm{~h}$ at room temperature. The PVDF membranes were first probed with primary antibodies (1:1,000 dilution) overnight at $4^{\circ} \mathrm{C}$, and then re-probed with a horseradish peroxidase-conjugated secondary antibody (1:1,000-1:2,000 dilution; Wuhan Boster Biological Technology, Ltd.) for $1.5 \mathrm{~h}$ at $37^{\circ} \mathrm{C}$. Antibodies against the following proteins were used: ERK1/2 (cat. no. 9102; 1:1,000; Cell Signaling Technology, Inc.), phosphorylated (p-)ERK1/2 (cat. no. 9101; 1:1,000; Cell Signaling Technology, Inc.), Akt (cat. no. AA326; 1:1,000; Beyotime Institute of Biotechnology), p-Akt (Ser473; cat. no. AA326; 1:1,000; Beyotime Institute of Biotechnology), STIP1 (cat.no. 4464; 1:1,000; Cell Signaling Technology, Inc.), matrix metalloproteinase (MMP)-2 (cat. no. sc-13594; 1:500; Santa Cruz Biotechnology, Inc.), MMP-9 (cat. no. sc-12759; 1:500; Santa Cruz Biotechnology, Inc.), tissue inhibitor of metalloproteinase (TIMP)-2 (cat. no. sc-21735; 1:500; Santa Cruz Biotechnology, Inc.), and GAPDH (cat. no. A00227; 1:1,000; Wuhan Boster Biological Technology, Ltd.,). After washing the PVDF membranes three times with TBS-T, chemiluminescence emission was visualized using a SuperSignal West Femto Substrate Trial kit (Thermo Fisher Scientific, Inc.) Each western blot image presented is from the same lysate and is representative of three separate experiments. The relative intensity of MMP-2, MMP-9 and TIMP-2 was normalized to the corresponding GAPDH intensity, and that of p-Akt and p-ERK1/2 was normalized to the corresponding total Akt and ERK1/2 intensity, respectively. The signal intensities of bands were quantified using ImageJ software 1.52a (National Institutes of Health).

Statistical analysis. Quantitative data are presented as the mean \pm standard error of the mean. Statistical analyses were performed using SPSS 15.0 statistics software (SPSS, Inc.). One-way ANOVA followed by Tukey's post hoc test for multiple comparisons was used to assess the differences among groups containing one variable, whereas a two-way ANOVA followed by the Bonferroni post hoc test was used to assess the differences among groups containing two variables. $\mathrm{P}<0.05$ was considered to indicate a statistically significant difference for all analyses.

\section{Results}

Isolation and identification of human osteosarcoma CD133+ CSCs. CD133+ cells were isolated via MACS from a population of MG63 cells in the logarithmic phase of growth, and then inoculated in culture bottles (Fig. 1A). When observed under a light microscope, CD133+ MG63 cells appeared irregular, fibroblast-like and had a large nucleus (Fig. 1B). When the cells were cultured in serum-free medium for 7-10 days, CD133+ osteosarcoma CSC spheres formed; these were characterized by a round shape, a regular border, and a cell number of $\geq 50$ per sphere (Fig. 1C). Compared with a previous study (19), microscopy indicated that $\mathrm{CD}_{133^{+}}$osteosarcoma CSC spheres did not vary greatly in size and shape, and closely resembled the spheres formed by MG63 cells; however the quantity of the former was larger than the latter.

$\mathrm{CD}_{133^{+}}$osteosarcoma CSCs have the ability to form secondary CSC spheres that are similar to the primary CSC spheres at a higher frequency. When $\mathrm{CD}_{133^{+}}$osteosarcoma
CSC spheres were cultured in serum-supplemented medium, the spheres adhered to the wall and produced new cancer cells (Fig. 1D).

Certain stem cell surface markers, such as CD44, CD105, CD133, and key characteristic molecules of pluripotent stem cells, such as Stro-1 and Nanog, are considered as markers of osteosarcoma CSCs $(12,13,18,19,22)$. Thus, CD44, CD105 and CD133 were selected as CSC markers for their identification by flow cytometry. Only $28.78 \pm 3.67 \%$ of MG63 cells were CD133 ${ }^{+}$, but the proportion in CSCs was $96.82 \pm 1.83 \%$ (Fig. 1E, F and K). Among the CD133 ${ }^{+} \mathrm{CSCs}$, $85.13 \pm 3.84 \%$ were $\mathrm{CD} 44^{+}$, compared with $47.77 \pm 6.60 \%$ for MG63 cells (Fig. 1G, H and K). For CD105, the percentages were $26.18 \pm 4.13$ and $3.46 \pm 0.44 \%$ in CSCs and MG63 cells, respectively (Fig. 1I-K).

Inhibition of STIP1 expression by siRNA. Following transfection of siRNAs targeting STIP1, efficiency was detected by fluorescence microscopy after $24 \mathrm{~h}$ (Fig. 2A and B); the images indicate that the transfection was successful. To evaluate whether the STIP1 siRNAs inhibited STIP1 expression, the levels of STIP1 mRNA and protein in the $\mathrm{CD} 133^{+}$osteosarcoma CSCs were analyzed using RT-qPCR and western blotting $48 \mathrm{~h}$ after transfection. Both the mRNA (Fig. 2C) and protein expression levels (Fig. 2D and E) of STIP1 were knocked down in the STIP1 siRNA-transfected CSCs compared with those observed in the NC siRNA-transfected and blank control (non-siRNA-transfected) groups, siRNA-643 group showed the best inhibition effect on STIP, siRNA-941 group showed a medium inhibition effect, and siRNA-1315 group showed the worst inhibition effect (Fig. 2C, D and E). In order to evaluate the biological effects of interfering STIP1 and explore the biological differences among different interference levels, siRNA-643 and siRNA-1315 were used in subsequent experiments.

Inhibition of cellmigration and invasion abilityfollowing STIP1 knockdown. To determine whether STIP1 knockdown affected cell proliferation, a CCK-8 assay was performed within $48 \mathrm{~h}$ of transfection. No significant changes in cell proliferation were observed between the NC and siRNA groups at 24 and $48 \mathrm{~h}$ (Fig. 3A). To examine the effect of STIP1 on the migration ability of $\mathrm{CD}_{133^{+}}$osteosarcoma CSCs, a wound-healing assay was performed, which demonstrated that the wound healing ability of STIP1 siRNA-643 transfected CSCs were weaker than those of the $\mathrm{NC}(\mathrm{P}<0.05)$ at $48 \mathrm{~h}($ Fig. 3B, D-I). To investigate the effect of STIP1 on the invasion capability of $\mathrm{CD}_{133}{ }^{+}$osteosarcoma CSCs, a Matrigel Transwell assay was carried out, which confirmed that, following STIP knockdown, the number of CSCs that invaded the lower chambers was significantly decreased compared with that observed in the NC group $(\mathrm{P}<0.05)$. Furthermore, the number of invading cells in the siRNA-643 group was lower than that reported in the siRNA-1315 group (Fig. 3C and M-O). Overall, these results suggest that STIP1 knockdown inhibits the migration and invasion capabilities of CD133+ osteosarcoma CSCs.

STIP1 knockdown affects the expression of MMPs. Based on the association between MMPs and invasion in cancer, changes in MMP-2 and MMP-9 expression levels were 

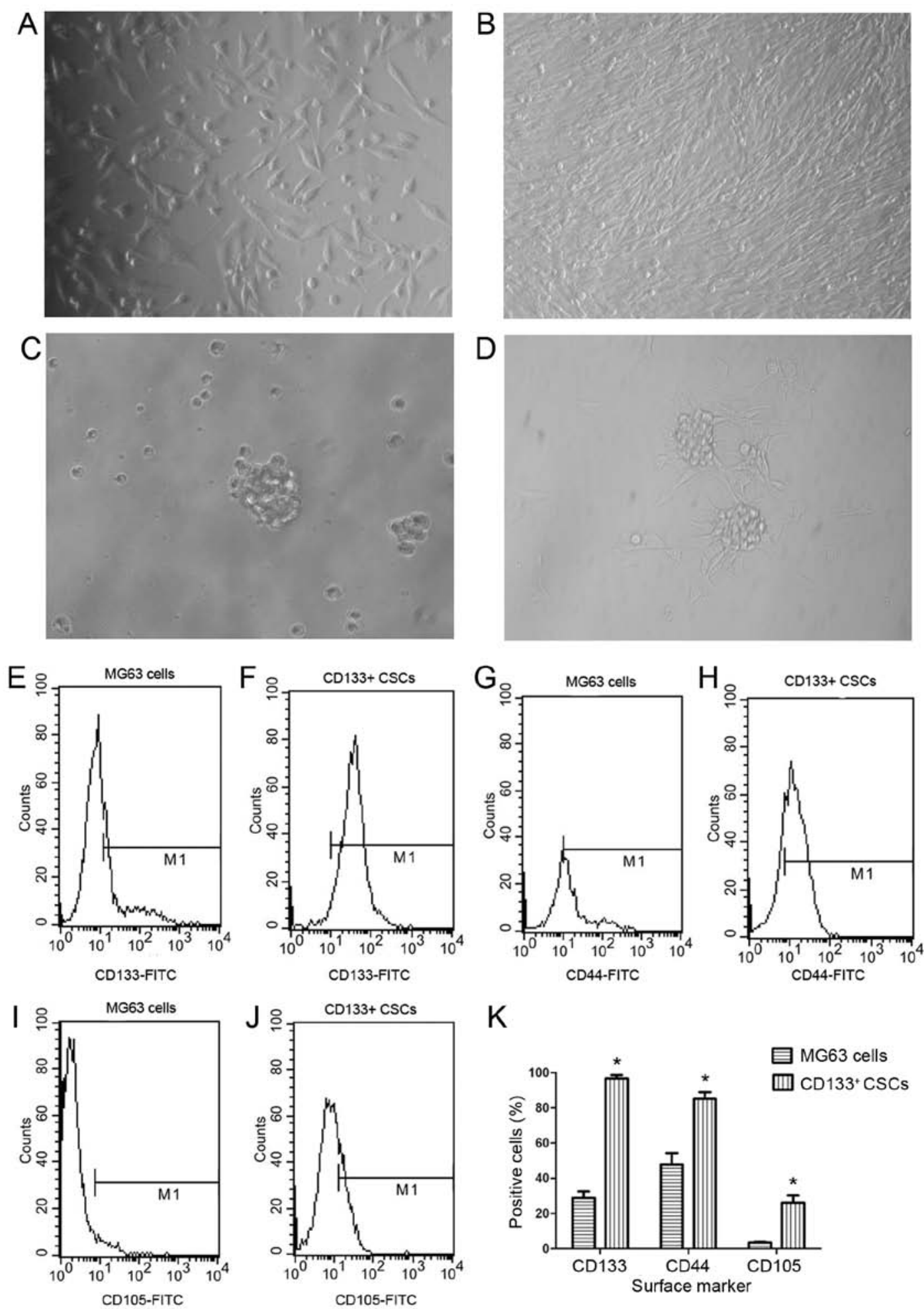

K

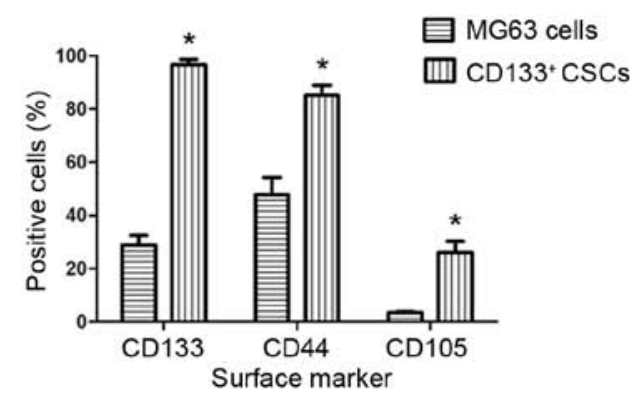

Figure 1. Isolation and identification of human osteosarcoma CD133 ${ }^{+}$CSCs. (A and B) CD133 ${ }^{+}$populations among MG63 cells (A) following overnight culture and (B) at the bottom of the culture dish. (C and D) CD133 ${ }^{+}$CSCs in (C) serum-free and (D) serum-supplemented media. Magnification, $\mathrm{x} 200$. (E and F) CD133 positive rate in (E) MG63 and (F) CSCs. (G and H) CD44-positive rate in (G) MG63 and (H) CSCs. (I and J) CD105-positive rate in (I) MG63 and (J) CSCs. (K) Positive rates of surface markers as assessed by flow cytometry. ${ }^{*} \mathrm{P}<0.05$ vs. the MG63 cells. CSCs, cancer stem-like cells.

examined in order to explore the effects of STIP1 on migration and invasion. The results demonstrated that the siRNA-643-transfected cells exhibited decreased MMP-2 and MMP-9 expression, and that TIMP-2 protein levels were significantly increased in both cell groups with STIP1 knockdown compared with the NC (Fig. 4A and B). These results were consistent with the results of the migration and invasion assays in $\mathrm{CD}_{133^{+}}$osteosarcoma CSCs.

STIP1 regulation of MMP expression may involve the PI3K/Akt and ERK1/2 signaling pathways. The present study aimed to determine the potential mechanism underlying the effect of 
A

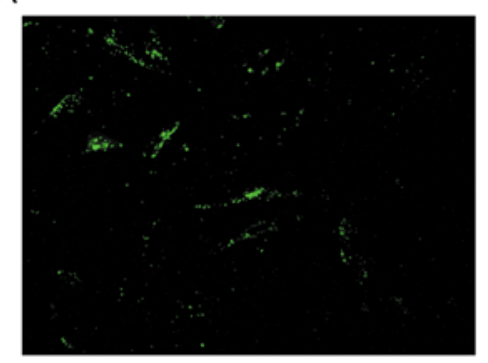

B

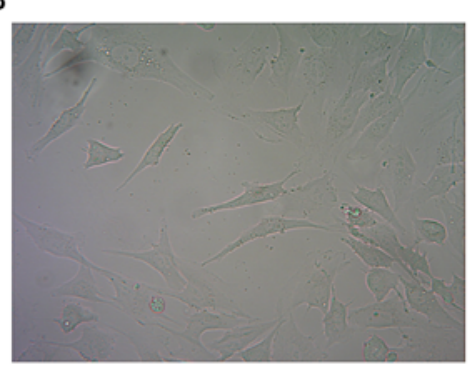

D

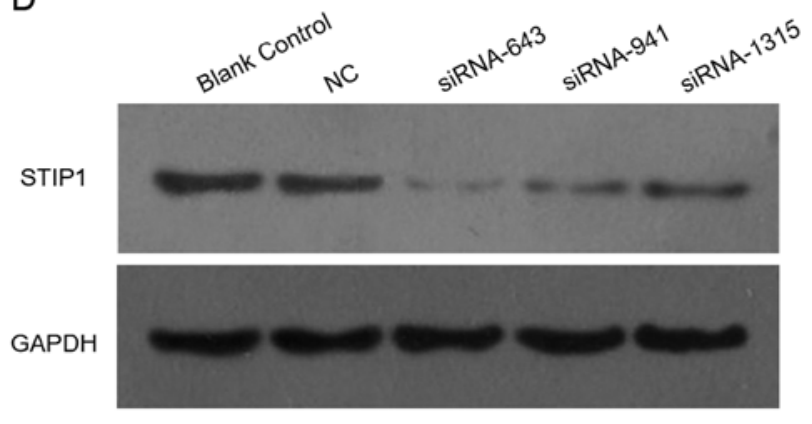

C

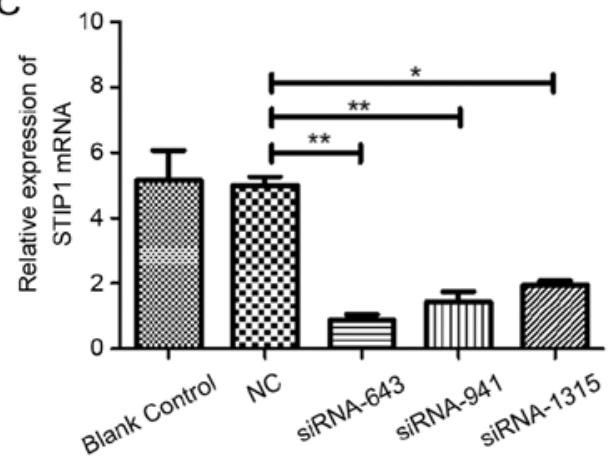

$E$

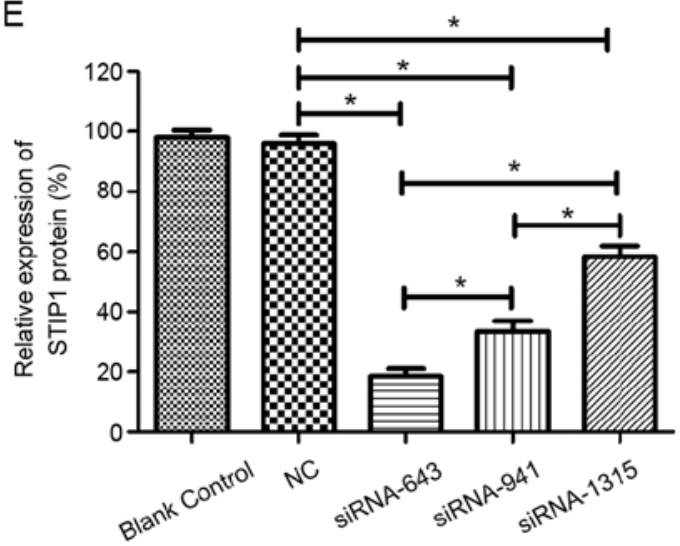

Figure 2. Transfection of STP1-siRNA in human osteosarcoma CD133+ cancer stem-like cells. (A and B) Representative images of transfection in CSCs assessed by (A) fluorescence and (B) light microscopy (magnification, x400). (C) STIP1-siRNA interference efficiency was assessed by reverse transcription-quantitative PCR. (D) STIP1-siRNA interference efficiency was assessed by western blotting and (E) the relative protein quantification was normalized to GAPDH levels. ${ }^{*} \mathrm{P}<0.05,{ }^{* *} \mathrm{P}<0.01$. STIP1, stress-induced phosphoprotein 1; siRNA, small interfering RNA.

STIP1 on CD133+ osteosarcoma CSC migration and invasion. Western blot analysis confirmed that p-Akt was downregulated following STIP1 knockdown, but the total Akt levels did not appear to be affected. In addition, the ratio of p-ERK1/2 to total ERK1/2 was significantly decreased in the siRNA-643 group compared with that in the NC (Fig. 4C and D). Thus, these results suggest that knockdown of STIP1 leads to a decrease in the levels of the phosphorylated forms of Akt and ERK1/2, and therefore, their activity.

\section{Discussion}

Based on current diagnostic guidelines and clinical experience, standard chemotherapy has been shown to provide significant benefits in terms of improving long-term survival (1). However, improvement in overall survival remains a challenge due to a large number of patients with osteosarcoma developing resistance to chemotherapeutic agents and undergoing metastasis (2). While a growing body of evidence supports the CSC theory, some researchers believe that tumors are derived from a single cancer-initiating cell with stem cell/cell-like properties, and that metastasis is attributable to this sub-population of cancer cells (16). Furthermore, osteosarcoma CSCs are responsible for the growth, metastasis and poor prognosis of osteosarcoma; agents that may inhibit the proliferation and differentiation of CSCs may provide new opportunities in the prevention of tumor recurrence and metastasis $(23,24)$. Previous studies have suggested that CSCs may be isolated from cell lines using MACS or the serum-free medium suspension cell sphere culture method $(18,19)$. According to characteristic surface markers and the self-renewal behavior of CSCs, the two methods were combined in the present study to isolate the osteosarcoma CSCs in order to improve the efficiency and purity of cell sorting.

CD133 is regarded as a marker of osteosarcoma CSCs and a potential target for drug delivery. Killing CD133+ osteosarcoma CSCs may significantly inhibit osteosarcoma growth $(14,25)$. CD133 could also be used as a sorting marker, as $\mathrm{CD}_{133^{+}}$osteosarcoma CSCs have high expression of stemness, metastasis and drug-resistance markers (13). Additionally, the results of the present study showed that there were higher levels of CD44, CD105 and CD133 expression in $\mathrm{CD}_{133^{+}}$osteosarcoma CSCs that underwent the serum-free medium culture sorting method, compared with MG63 cells, and suggest that the combined sorting method can be used as an efficient tool for CSC isolation.

Previous studies have demonstrated that STIP1 could serve as a novel biomarker for poor prognosis and advanced cancer progression in patients with malignant tumors, including ovarian cancer, hepatocellular carcinoma, esophageal squamous cell carcinoma, papillary thyroid carcinoma and gastric cancer $(7,26)$. Cell migration and invasion are important malignant characteristics in cancer metastasis (27). It has been reported that STIP1 may be associated with tumor metastasis (7). Iglesia et al (28) found that glioblastoma stem-like cells have high expression levels of STIP1, 
A

B
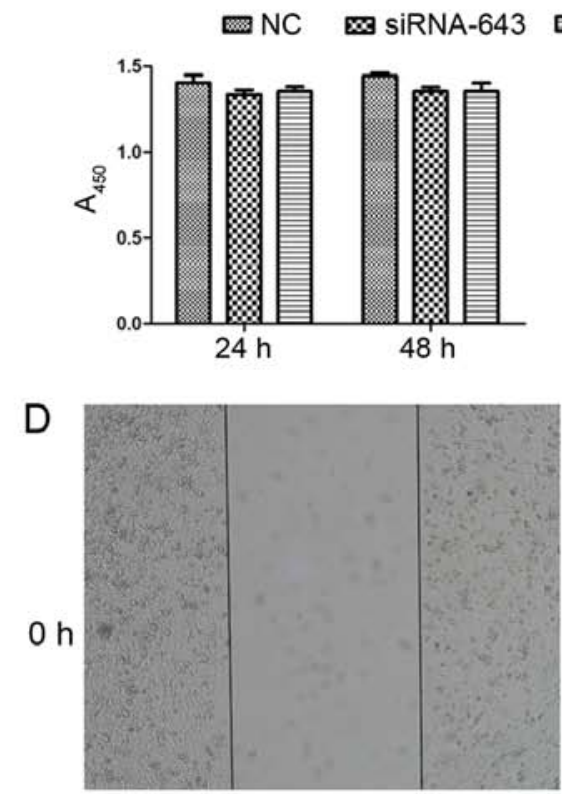

G

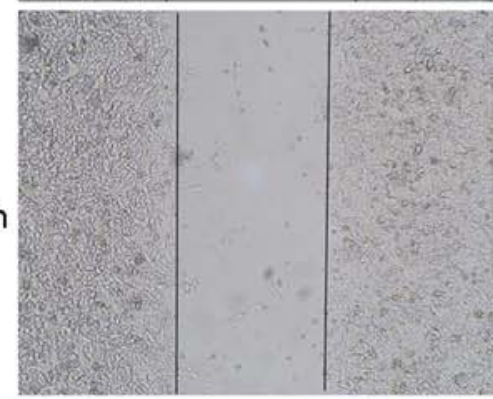

$\mathrm{J}$

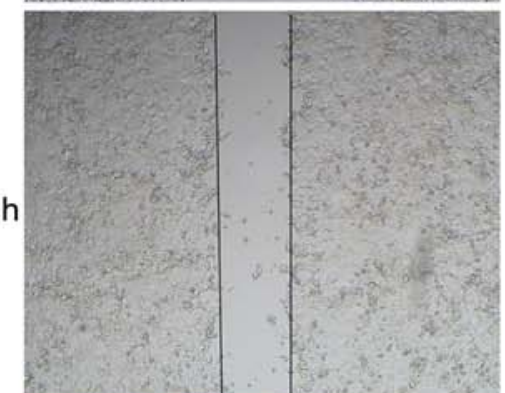

M

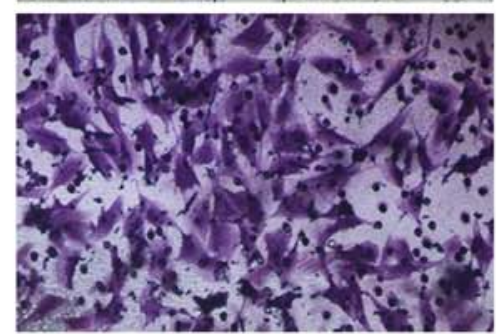

NC
A-1315
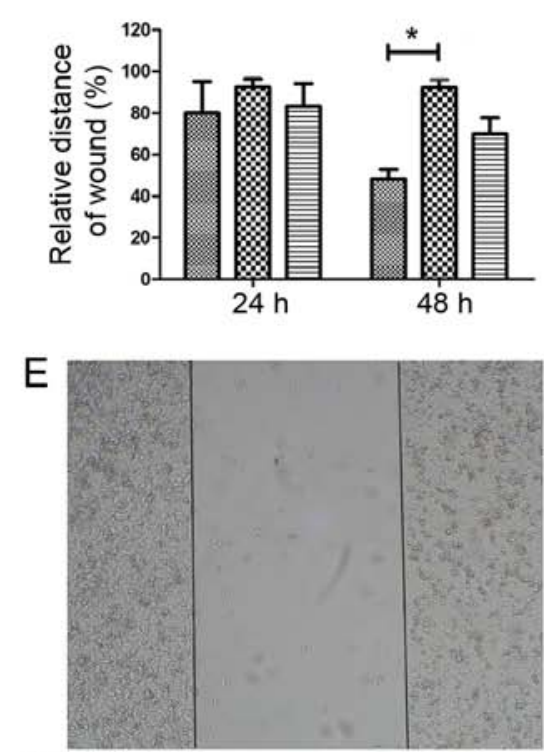

$\mathrm{H}$

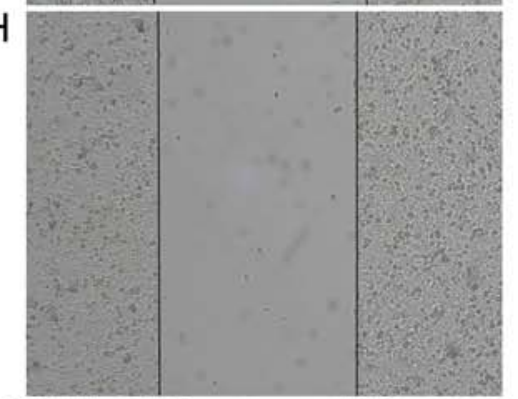

K

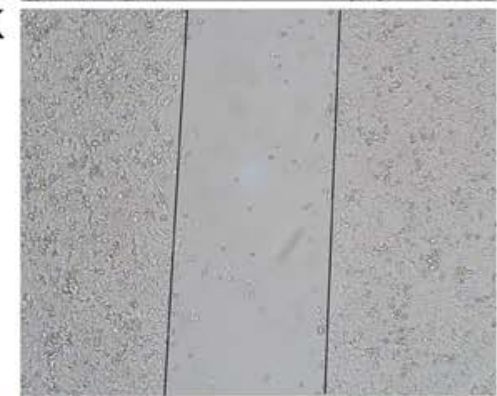

$\mathrm{N}$

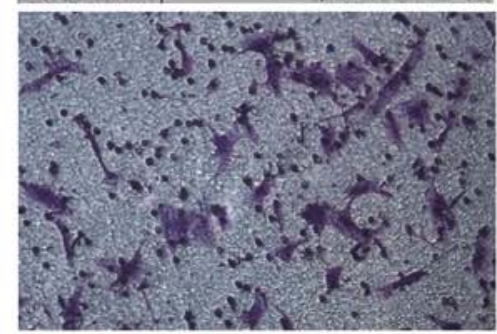

siRNA-643
C

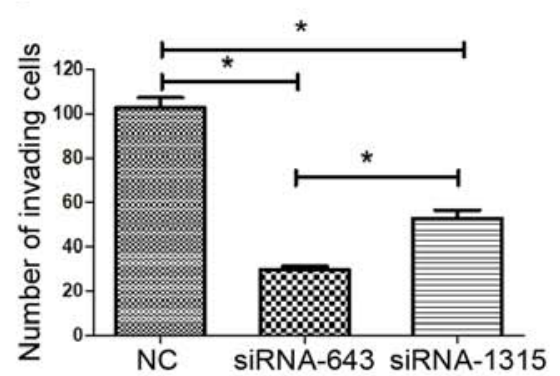

$\mathrm{F}$

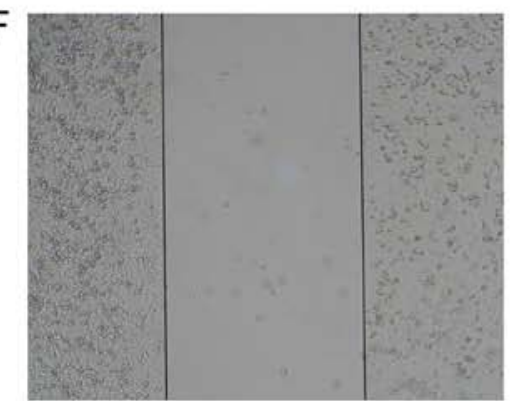

I

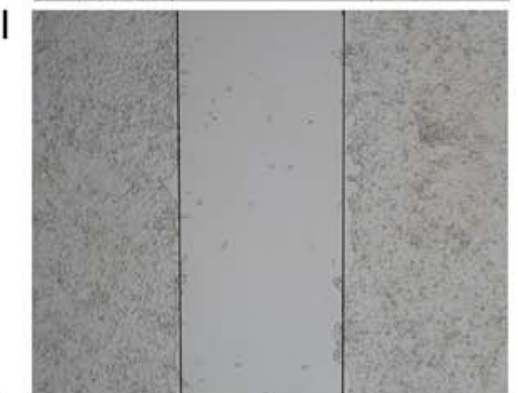

$\mathrm{L}$
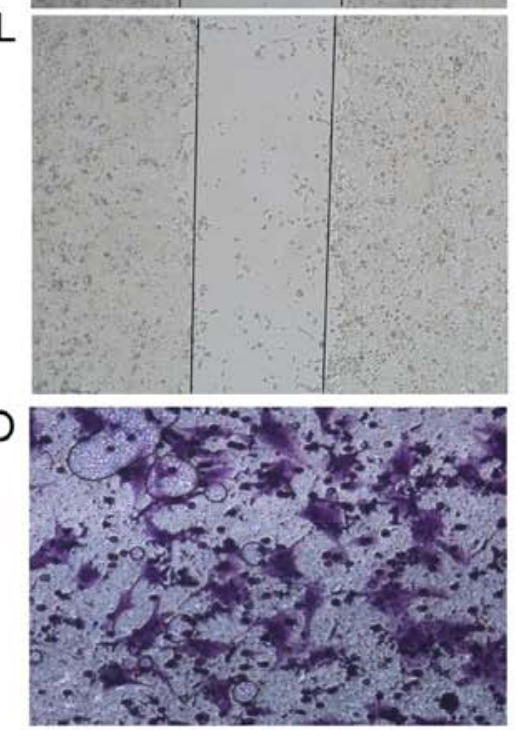

SiRNA-1315

Figure 3. Effects of STIP1 knockdown on cell proliferation, migration and invasion of CD133+ cancer stem-like cells. (A) Cell proliferation assay results following transfection with STIP1-siRNA and siRNA NC. (B) Quantification of the results of the wound-healing assay following 24 and $48 \mathrm{~h}$. (C) Matrigel invasion assay results. Representative wound-healing assay images at $0 \mathrm{~h}$ for cells transfected with (D) siRNA NC, (E) siRNA-643 and (F) siRNA-1315; at $24 \mathrm{~h}$ for cells transfected with (G) siRNA NC, (H) siRNA-643 and (I) siRNA-1315; and at $48 \mathrm{~h}$ for cells transfected with (J) siRNA NC, (K) siRNA-643 and (L) siRNA-1315. Representative images of the Matrigel invasion assay for cells transfected with (M) siRNA NC, (N) siRNA-643 and (O) siRNA-1315. Magnification, $x 100 .{ }^{*} \mathrm{P}<0.05$. STIP1, stress-induced phosphoprotein 1; siRNA, small interfering RNA; NC, negative control.

that STIP1 is able to modulate glioblastoma stem-like cell proliferation and self-renewal, and that STIP1-knockdown affects proliferative and tumorigenic capacity. It is well known that MMPs play a key role in cell migration and invasion, in that cell invasion involves the degradation of the basement membrane extracellular matrix (27). Walsh et al (8) showed that STIP1 knockdown was able to inhibit invasion in pancreatic cancer cells, and that this inhibition may be induced via 
A
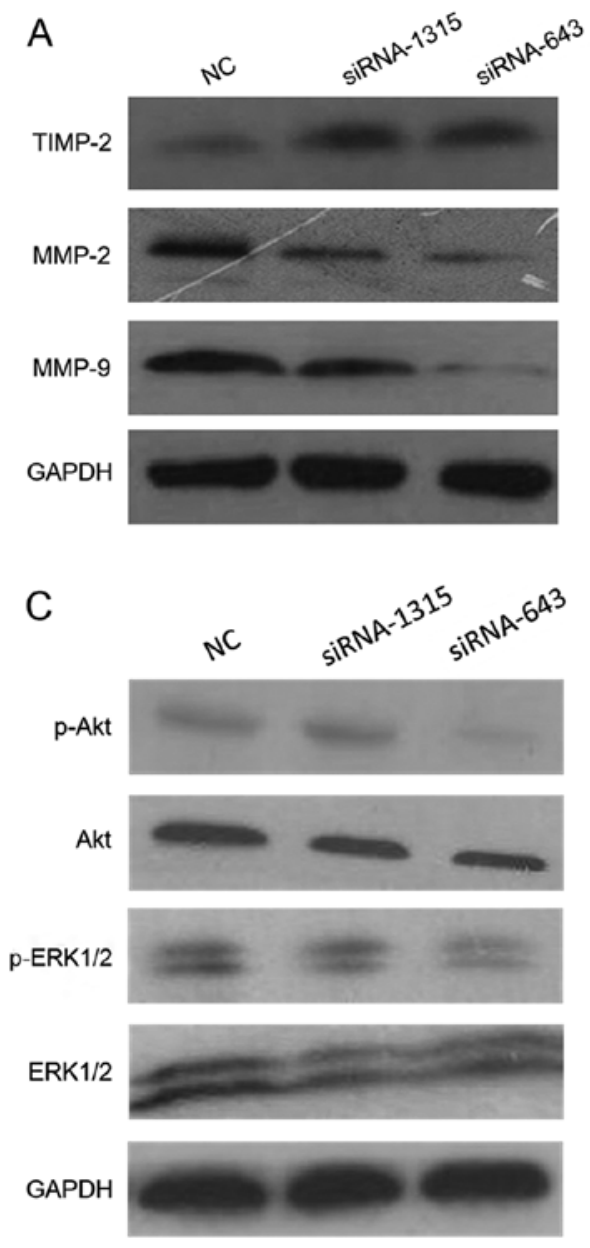

B

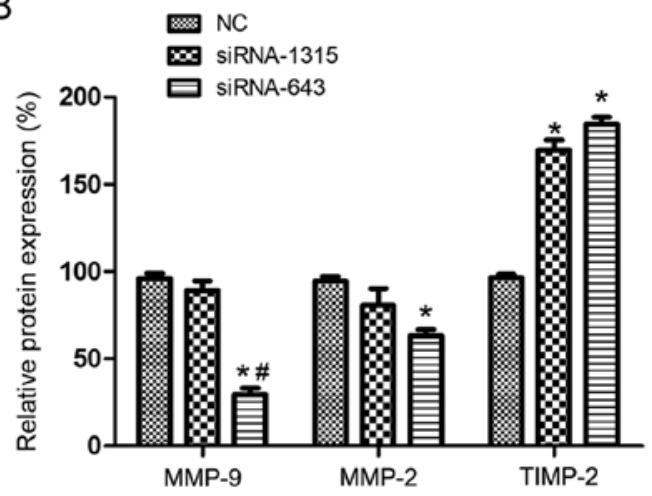

D
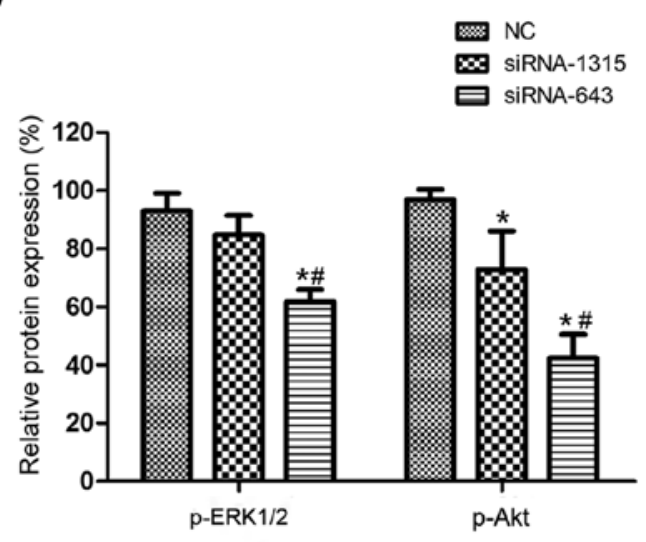

Figure 4. Protein expression changes following STIP1 knockdown in CD133 ${ }^{+}$cancer stem-like cells. (A) Representative western blot images and (B) relative quantification of TIMP-2, MMP-2 and MMP-9 proteins, normalized to GAPDH levels. (C) Representative western blot images and (D) relative quantification of p-Akt and p-ERK1/2 normalized to the respective total protein levels. ${ }^{*} \mathrm{P}<0.05$ vs. NC; ${ }^{\text {P }}<0.05$ vs. siRNA-1315 group. TIMP-2, tissue inhibitor of metalloproteinase-2; p-Akt, phosphorylated Akt; MMP, matrix metalloproteinase; NC, negative control; siRNA, small interfering RNA.

the downregulation of MMP-2. Furthermore, the same study revealed that STIP1-siRNA did not alter levels of HSP70 or HSP90 expression, whereas the expression levels of the HSP90 client proteins such as HER2, Bcr-Abl, and proto-oncogenes c-MET and v-Src were decreased with STIP1-siRNA transfection. In addition, STIP1 was able to act as a transcription factor and bind to the MMP-9 promoter to enhance MMP-9 transcriptional expression (29). In the present study, it was shown that STIP1 knockdown was able to inhibit CD133+ osteosarcoma CSC migration and invasion, but did not directly affect the ability of the cells to proliferate for $48 \mathrm{~h}$, in agreement with studies by Huang et al (27) and Walsh et al (8). Moreover, the results of the present study showed that STIP1 knockdown reduced the expression of MMP-2 and MMP-9, and promoted the expression of TIMP-2.

To further the understanding of the molecular mechanisms by which STIP1 knockdown inhibits CD133+ osteosarcoma CSC migration and invasion, western blotting was used to investigate the association of STIP1 with the ERK1/2 and PI3K/Akt pathways. The PI3K/Akt signaling pathway is important in regulating the processes of cancer development, including tumor formation, progression, multi-drug resistance, tumor invasion and metastasis (19). Erlich et al (30) reported that STIP1 promoted glioma proliferation through the MAPK and PI3K pathways. Other signaling pathways, such as ERK1/2, Wnt/ $\beta$-catenin, STAT3 and JAK2, are triggered by STIP1 in various types of cancer $(7,31)$. The results of the present study suggest that STIP1 knockdown may inhibit Akt and ERK1/2 phosphorylation, and subsequently inhibit the activity of the PI3K/Akt and ERK1/2 pathways, in agreement with the results reported by Wang et al (29).

In summary, the present study identified an association between STIP1 and osteosarcoma CSC invasion, as well as its potential molecular mechanisms. A better understanding of the role of STIP1 in osteosarcoma could facilitate the development of molecular targeted therapies able to reduce the metastasis rate and improve overall patient survival.

\section{Acknowledgements}

No applicable.

\section{Funding}

This work was supported by the Hubei Province Health and Family Planning Scientific Research Project (grant no. WJ2017M058). 


\section{Availability of data and materials}

The datasets used and/or analyzed in the current study are available from the corresponding author on reasonable request.

\section{Authors' contributions}

JHW, YL and QL performed the experiments. JHW edited and verified the manuscript. CG and HHX designed the study and wrote the first draft of the manuscript. FJG provided the MG63 cells and performed in vitro experiments. XZ revised the first draft of the article and repeated some of the experiments. MSZ and HQ assisted with the statistics and survival data analysis. TFC performed the experiments and participated in writing and revising the manuscript. CG, JHW and HHX helped design the figures and tables. All authors read and approved the final manuscript.

\section{Ethics approval and consent to participate}

Not applicable.

\section{Patient consent for publication}

Not applicable.

\section{Competing interests}

The authors declare that they have no competing interests.

\section{References}

1. Heare T, Hensley MA and Dell'Orfano S: Bone tumors: Osteosarcoma and Ewing's sarcoma. Curr Opin Pediatr 21: 365-372, 2009

2. Xiao X, Wang W, Li Y, Yang D, Li X, Shen C, Liu Y, Ke X, Guo S and Guo Z: HSP90AA1-mediated autophagy promotes drug resistance in osteosarcoma. J Exp Clin Cancer Res 37: 201, 2018

3. Ellis RJ: Molecular chaperones: Assisting assembly in addition to folding. Trends Biochem Sci 31: 395-401, 2006.

4. Wang Y and McAlpine SR: Regulating the cytoprotective response in cancer cells using simultaneous inhibition of Hsp90 and Hsp70. Org Biomol Chem 13: 2108-2116, 2015.

5. Longshaw VM, Baxter M, Prewitz M and Blatch GL: Knockdown of the co-chaperone Hop promotes extranuclear accumulation of Stat3 in mouse embryonic stem cells. Eur J Cell Biol 88: 153-166, 2009.

6. Longshaw VM, Chapple JP, Balda MS, Cheetham ME and Blatch GL: Nuclear translocation of the Hsp70/Hsp90 organizing protein $\mathrm{mSTI} 1$ is regulated by cell cycle kinases. J Cell Sci 117 (Pt 5): 701-710, 2004.

7. Zhai E, Liang W, Lin Y, Huang L, He X, Cai S, Chen J, Zhang N, Li J, Zhang Q, et al: HSP70/HSP90-Organizing protein contributes to gastric cancer progression in an autocrine fashion and predicts poor survival in gastric cancer. Cell Physiol Biochem 47: 879-892, 2018

8. Walsh N, Larkin A,Swan N, Conlon K, Dowling P, McDermott R and Clynes M: RNAi knockdown of Hop (Hsp70/Hsp90 organising protein) decreases invasion via MMP-2 down regulation. Cancer Lett 306: 180-189, 2011.

9. Baindur-Hudson S, Edkins AL and Blatch GL: Hsp70/Hsp90 organising protein (hop): Beyond interactions with chaperones and prion proteins. Subcell Biochem 78: 69-90, 2015.

10. Liu B, Ma W, Jha RK and Gurung K: Cancer stem cells in osteosarcoma: Recent progress and perspective. Acta Oncol 50: 1142-1150, 2011

11. Yang M, Yan M, Zhang R, Li J and Luo Z: Side population cells isolated from human osteosarcoma are enriched with tumor-initiating cells. Cancer Sci 102: 1774-1781, 2011.
12. Gibbs CP, Kukekov VG, Reith JD, Tchigrinova O, Suslov ON, Scott EW, Ghivizzani SC, Ignatova TN and Steindler DA: Stem-like cells in bone sarcomas: Implications for tumorigenesis. Neoplasia 7: 967-976, 2005.

13. Koka P, Mundre RS, Rangarajan R, Chandramohan Y, Subramanian RK and Dhanasekaran A: Uncoupling Warburg effect and stemness in CD133 $3^{\text {+ve }}$ cancer stem cells from Saos-2 (osteosarcoma) cell line under hypoxia. Mol Biol Rep 45: $1653-1662,2018$

14. Ni M, Xiong M, Zhang X, Cai G, Chen H, Zeng Q and Yu Z: Poly(lactic-co-glycolic acid) nanoparticles conjugated with CD133 aptamers for targeted salinomycin delivery to $\mathrm{CD} 133^{+}$ osteosarcoma cancer stem cells. Int J Nanomedicine 10: 2537-2554, 2015

15. Yang J, Guo W, Wang L, Yu L, Mei H, Fang S, Ji P, Liu Y, Liu G and Song Q: Cisplatin-resistant osteosarcoma cells possess cancer stem cell properties in a mouse model. Oncol Lett 12: 2599-2605, 2016

16. Brown HK, Tellez-Gabriel M and Heymann D: Cancer stem cells in osteosarcoma. Cancer Lett 386: 189-195, 2017.

17. Li K, Li X, Tian J, Wang H, Pan J and Li J: Downregulation of DNA-PKcs suppresses P-gp expression via inhibition of the Akt/NF-KB pathway in CD133-positive osteosarcoma MG-63 cells. Oncol Rep 36: 1973-1980, 2016.

18. Gong C, Liao H, Guo F, Qin L and Qi J: Implication of expression of Nanog in prostate cancer cells and their stem cells. J Huazhong Univ Sci Technolog Med Sci 32: 242-246, 2012.

19. Gong C, Liao H, Wang J, Lin Y, Qi J, Qin L, Tian LQ and Guo FJ: LY294002 induces G0/G1 cell cycle arrest and apoptosis of cancer stem-like cells from human osteosarcoma via down-regulation of PI3K activity. Asian Pac J Cancer Prev 13: 3103-3107, 2012.

20. Livak KJ and Schmittgen TD: Analysis of relative gene expression data using real-time quantitative PCR and the 2(-Delta Delta C(T)) method. Methods 25: 402-408, 2001.

21. Li J, Sun X, Wang Z, Chen L, Li D, Zhou J and Liu M: Regulation of vascular endothelial cell polarization and migration by Hsp70/Hsp90-organizing protein. PLoS One 7: e36389, 2012.

22. Abarrategi A, Tornin J, Martinez-Cruzado L, Hamilton A, Martinez-Campos E, Rodrigo JP, González MV, Baldini N, Garcia-Castro Jand RodriguezR: Osteosarcoma: Cells-of-Origin, cancer stem cells, and targeted therapies. Stem Cells Int 2016: 3631764, 2016.

23. Qi XT, Li YL, Zhang YQ, Xu T, Lu B, Fang L, Gao JQ, Yu LS, Zhu DF, Yang B, et al: KLF4 functions as an oncogene in promoting cancer stem cell-like characteristics in osteosarcoma cells. Acta Pharmacol Sin 40: 546-555, 2019.

24. Chang Y, Zhao Y, Zhan H, Wei X, Liu T and Zheng B: Bufalin inhibits the differentiation and proliferation of human osteosarcoma cell line hMG63-derived cancer stem cells. Tumour Biol 35: 1075-1082, 2014.

25. Chen F, Zeng Y, Qi X, Chen Y, Ge Z, Jiang Z, Zhang X, Dong $\mathrm{Y}, \mathrm{Chen} \mathrm{H}$ and $\mathrm{Yu} \mathrm{Z}$ : Targeted salinomycin delivery with EGFR and CD133 aptamers based dual-ligand lipid-polymer nanoparticles to both osteosarcoma cells and cancer stem cells. Nanomedicine 14: 2115-2127, 2018.

26. Xu YW, Liu CT, Huang XY, Huang LS, Luo YH, Hong CQ, Guo HP, Xu LY, Peng YH and Li EM: Serum Autoantibodies against STIP1 as a potential biomarker in the diagnosis of esophageal squamous cell carcinoma. Dis Markers 2017: 5384091, 2017.

27. Huang L, Zhai E, Cai S, Lin Y, Liao J, Jin H, Peng S, Xu L, Chen $M$ and Zeng Z: Stress-inducible Protein-1 promotes metastasis of gastric cancer via Wnt $/ \beta$-catenin signaling pathway. J Exp Clin Cancer Res 37: 6, 2018.

28. Iglesia RP, Prado MB, Cruz L, Martins VR, Santos TG and Lopes MH: Engagement of cellular prion protein with the co-chaperone Hsp70/90 organizing protein regulates the proliferation of glioblastoma stem-like cells. Stem Cell Res Ther 8: 76, 2017.

29. Wang HS, Tsai CL, Chang PY, Chao A, Wu RC, Chen SH, Wang CJ, Yen CF, Lee YS and Wang TH: Positive associations between upregulated levels of stress-induced phosphoprotein 1 and matrix metalloproteinase-9 in endometriosis/adenomyosis. PLoS One 13: e190573, 2018

30. Erlich RB, Kahn SA, Lima FR, Muras AG, Martins RA, Linden R, Chiarini LB, Martins VR and Moura Neto V: STI1 promotes glioma proliferation through MAPK and PI3K pathways. Glia 55: 1690-1698, 2007.

31. Tsai CL, Chao A, Jung SM, Tsai CN, Lin CY, Chen SH, Sue SC, Wang TH, Wang HS and Lai CH: Stress-induced phosphoprotein-1 maintains the stability of JAK2 in cancer cells. Oncotarget 7: 50548-50563, 2016. 